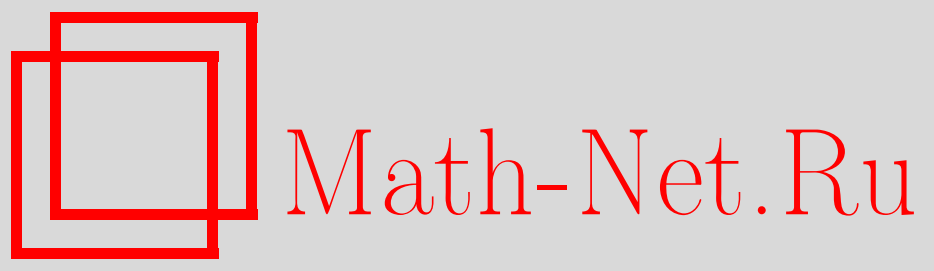

С. Н. Лакаев, Ш. У. Алладустов, Положительность собственных значений двухчастичного оператора Шредингера на решетке, ТМФ, 2014, том 178, номер 3, 390-402

DOI: https://doi.org/10.4213/tmf8544

Использование Общероссийского математического портала Math-Net.Ru подразумевает, что вы прочитали и согласны с пользовательским соглашением http://www . mathnet.ru/rus/agreement

Параметры загрузки:

IP : 3.85 .5 .30

26 апреля 2023 г., 15:15:46

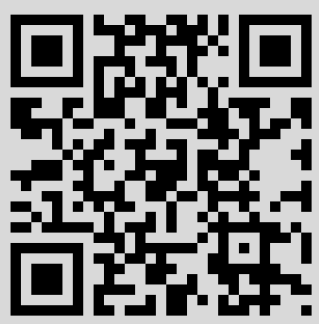




\title{
ФИЗИКА
}

Том 178, № 3

март, 2014

\section{ПОЛОЖИТЕЛЬНОСТЬ СОБСТВЕННЫХ ЗНАЧЕНИЙ ДВУХЧАСТИЧНОГО ОПЕРАТОРА ШРЕДИНГЕРА НА РЕШЕТКЕ}

\begin{abstract}
Рассмотрено семейство $H(k)$ двухчастичных дискретных операторов Шредингера, зависящих от квазиимпульса системы двух частиц $k \in \mathbb{T}^{d}$, здесь $\mathbb{T}^{d}-$ $d$-мерный тор. Это семейство операторов ассоциировано с гамильтонианом системы двух произвольных частиц на $d$-мерной решетке $\mathbb{Z}^{d}, d \geqslant 3$, которые взаимодействуют с помощью парного короткодействующего потенциала притяжения. Доказано, что собственные значения оператора Шредингера $H(k)$, лежащие ниже левой границы существенного спектра, положительны при всех ненулевых значениях квазиимпульса $k \in \mathbb{T}^{d}$, если оператор $H(0)$ неотрицательный. Установлен аналогичный результат для собственных значений оператора Шредингера $H_{+}(k), k \in \mathbb{T}^{d}$, соответствующего системе двух частиц с отталкивающим взаимодействием.
\end{abstract}

Ключевые слова: дискретный оператор Шредингера, квазиимпульс системы, гамильтониан, отталкивающее взаимодействие, виртуальный уровень, собственное значение, решетка.

DOI: $10.4231 / \operatorname{tmf} 8544$

\section{1. ВВЕДЕНИЕ}

В настоящей работе рассматривается семейство дискретных операторов Шредингера $H(k), k \in \mathbb{T}^{d}$, где $\mathbb{T}^{d}-d$-мерный тор, ассоциированное с гамильтонианом системы двух произвольных квантовых частиц, которые движутся на $d$-мерной решетке $\mathbb{Z}^{d}$ и взаимодействуют с помощью парного короткодействующего потенциала притяжения $\hat{v}$.

В физике устойчивые сложные объекты обычно образуются в результате действия сил притяжения, которые позволяют составным частям уменьшить энергию при их связывании. Математически это явление описывается непрерывным оператором Шредингера, который может иметь связанное состояние с отрицательной энергией. Дискретный оператор Шредингера является ограниченным, и его непрерывный спектр заполняет некоторый отрезок, поэтому такой оператор может иметь

* Самаркандский государственный университет, Самарканд, Узбекистан. E-mail: slakaev@math.ucdavis.edu,sh.alladustov@gmail.com 
связанное состояние, энергия которого лежит правее порога непрерывного спектра [1]. Этот результат является теоретическим обоснованием экспериментального наблюдения, состоящего в том, что в упорядоченных средах как с периодическим потенциалом, так и в отсутствии рассеяния устойчивые сложные объекты могут существовать даже в случае отталкивающих взаимодействий [2]. Для операторов Шредингера $H(k)=H_{0}(k)-V, k \in \mathbb{T}^{d}$, которые ассоциированы с гамильтонианом $H$ системы двух бозонов на решетке $\mathbb{Z}^{d}$, взаимодействующих с помощью контактного потенциала притяжения $-V$, было доказано [3], [4], что если при значении квазиимпульса $k=0$ оператор $H(0)=H_{0}(0)-V$ является неотрицательным, то при любом $k \neq 0$ оператор $H(k)=H_{0}(k)-V$ (строго) положителен.

Для некоторого, но важного для применений класса операторов Шредингера $H(k)=H_{0}(k)-V, k \in \mathbb{T}^{d}$, ассоциированных с гамильтонианом $H$ системы двух одинаковых частиц (бозонов или фермионов), которые взаимодействуют с помощью парного короткодействующего потенциала притяжения $-V$, было доказано [5], что если при $k=0$ оператор $H(0)=H_{0}(0)-V$ является неотрицательным, то при любом значении квазиимпульса $k \neq 0$ оператор $H(k)=H_{0}(k)-V$ также неотрицательный.

В настоящей работе мы изучаем, применяя предельный принцип Бирмана-Швингера, зависимость собственных значений оператора $H(k)=H_{0}(k)-V$ от квазиимпульса системы $k \in \mathbb{T}^{d}$. А именно, мы доказываем результат, более сильный, чем в работе [5], состоящий в том, что если оператор $H(0)=H_{0}(0)-V$ неотрицателен, то при любом ненулевом значении квазиимпульса $k$ оператор $H(k)=H_{0}(k)-V$ является (строго) положительным (см. следствие 1).

Из этого утверждения мы получаем, что если оператор $H(0)$ неотрицательный, то при любом ненулевом значении квазиимпульса $k$ все собственные значения оператора $H(k)$, лежащие левее порога существенного спектра, положительны. Последнее влечет следующий факт для трехчастичного дискретного оператора Шредингера $\mathbf{H}(K), K \in \mathbb{T}^{3}$, который ассоциирован с гамильтонианом $\mathbf{H}$ системы трех частиц, движущихся на решетке $\mathbb{Z}^{3}$ и взаимодействующих с помощью парных короткодействующих потенциалов притяжения: трехчастичный оператор Шредингера $\mathbf{H}(0)$ имеет бесконечное число собственных значений (эффект Ефимова), однако при любом ненулевом значении квазиимпульса $K \in \mathbb{T}^{3}$ системы оператор $\mathbf{H}(K)$ имеет только конечное число собственных значений [3], [4], [6], [7].

Результаты настоящей работы характерны только для дискретных операторов Шредингера и не имеет аналогов для непрерывных операторов Шредингера, у которых спектр не зависит от полного импульса системы $k \in \mathbb{R}^{d}$.

\section{2. ДВУХЧАСТИЧНЫЙ ГАМИЛЬТОНИАН $\widehat{H}$ И ДИСКРЕТНЫЙ ОПЕРАТОР ШРЕДИНГЕРА $H(K)$}

Гамильтониан $\widehat{H}$ системы двух произвольных частиц, взаимодействующих с помощью парного короткодействующего потенциала $\widehat{V}$, в координатном представлении обычно ассоциируется с ограниченным самосопряженным оператором $\widehat{H}=\widehat{H}_{0}-\widehat{V}$, действующим в гильбертовом пространстве $\ell^{2}\left(\left(\mathbb{Z}^{d}\right)^{2}\right)$. Здесь гамильтониан $\widehat{H}_{0}$ системы двух произвольных свободных (невзаимодействующих) частиц действует в пространстве $\ell^{2}\left(\left(\mathbb{Z}^{d}\right)^{2}\right)$ по формуле

$$
\left(\widehat{H}_{0} \hat{\psi}\right)\left(x_{1}, x_{2}\right)=\sum_{s \in \mathbb{Z}^{d}}\left[\hat{\varepsilon}_{1}(s) \hat{\psi}\left(x_{1}+s, x_{2}\right)+\hat{\varepsilon}_{2}(s) \hat{\psi}\left(x_{1}, x_{2}+s\right)\right], \quad \hat{\psi} \in \ell^{2}\left(\left(\mathbb{Z}^{d}\right)^{2}\right),
$$


где функции $\hat{\varepsilon}_{j}(s), j=1,2$, определены на $\mathbb{Z}^{d}$ и принадлежат пространству $\ell^{2}\left(\mathbb{Z}^{d}\right)$. Предположим также, что выполняется условие самосопряженности оператора $\widehat{H}_{0}$ :

$$
\hat{\varepsilon}_{j}(s)=\overline{\hat{\varepsilon}_{j}(-s)}, \quad s \in \mathbb{Z}^{d}, \quad j=1,2 .
$$

Функции $\varepsilon_{1}(s)$ и $\varepsilon_{2}(s)$, заданные как ряды Фурье

$$
\varepsilon_{j}(p)=\sum_{s \in \mathbb{Z}^{d}} \hat{\varepsilon}_{j}(s) e^{i(p, s)}, \quad p \in \mathbb{T}^{d}, \quad j=1,2,
$$

являются вещественнозначными функциями на $\mathbb{T}^{d}$.

Оператор умножения $\widehat{V}$ определен в $\ell^{2}\left(\left(\mathbb{Z}^{d}\right)^{2}\right)$ как оператор умножения на функцию:

$$
(\widehat{V} \hat{\psi})\left(x_{1}, x_{2}\right)=\hat{v}\left(x_{1}-x_{2}\right) \hat{\psi}\left(x_{1}, x_{2}\right), \quad \hat{\psi} \in \ell^{2}\left(\left(\mathbb{Z}^{d}\right)^{2}\right),
$$

где $\hat{v}=\{\hat{v}(s)\}_{s \in \mathbb{Z}^{d}}$ - ограниченная последовательность вещественных чисел. Предположим, что

$$
\lim _{x \rightarrow \infty} \hat{v}(x)=0
$$

В этом случае $\widehat{V}$ является компактным оператором.

Пусть $\mathbb{T}^{d}-d$-мерный тор, т. е. куб $(-\pi, \pi]^{d}$ с соответствующим отождествлением противоположных граней. Он рассматривается как абелева группа, в которой операции сложения и умножения на вещественное число введены как операции сложения и умножения на вещественное число в $\mathbb{R}^{d}$ по модулю $(2 \pi \mathbb{Z})^{d}$. Обозначим через $d \eta$ меру Хаара на торе $\mathbb{T}^{d}$. Пусть $L_{2}\left(\left(\mathbb{T}^{d}\right)^{2}, d \eta \times d \eta\right)$ - гильбертово пространство квадратично-интегрируемых функций, определенных на $\left(\mathbb{T}^{d}\right)^{2}$.

Переход в импульсное представление гамильтониана $\widehat{H}$ осуществляется с помощью преобразования Фурье

$$
\left.\mathcal{F}: L_{2}\left(\mathbb{T}^{d}\right)^{2}\right) \rightarrow \ell^{2}\left(\left(\mathbb{Z}^{d}\right)^{2}\right)
$$

В импульсном представлении полный двухчастичный гамильтониан $H=\mathcal{F} \widehat{H} \mathcal{F}^{-1}$ системы двух произвольных частиц действует в гильбертовом пространстве $L_{2}\left(\left(\mathbb{T}^{d}\right)^{2}\right)$ как ограниченный самосопряженный оператор $H=H_{0}-V$ [8], где $H_{0}$ - оператор умножения на функцию,

$$
\left(H_{0} f\right)\left(k_{1}, k_{2}\right)=\left(\varepsilon_{1}\left(k_{1}\right)+\varepsilon_{2}\left(k_{2}\right)\right) f\left(k_{1}, k_{2}\right), \quad f \in L_{2}\left(\left(\mathbb{T}^{d}\right)^{2}\right),
$$

a $V$ - оператор частичного интегрирования,

$$
(V f)\left(k_{1}, k_{2}\right)=\int_{\left(\mathbb{T}^{d}\right)^{2}} \delta\left(k_{1}+k_{2}-q_{1}-q_{2}\right) v\left(q_{1}-q_{2}\right) f\left(q_{1}, q_{2}\right) d \eta \times d \eta, \quad f \in L_{2}\left(\left(\mathbb{T}^{d}\right)^{2}\right),
$$

где

$$
v(p)=(2 \pi)^{-3 / 2} \sum_{s \in \mathbb{Z}^{d}} \hat{v}(s) e^{i(p, s)}
$$

и $\delta(k)$ обозначает дельта-функцию Дирака. 
Разложение гильбертова пространства $L_{2}\left(\left(\mathbb{T}^{d}\right)^{2}\right)$ как прямой интеграл,

$$
L_{2}\left(\left(\mathbb{T}^{d}\right)^{2}\right)=\int_{k \in \mathbb{T}^{d}} \oplus L_{2}\left(\mathbb{T}^{d}\right) d \eta
$$

позволяет записать разложение оператора $H$ в следующий прямой интеграл [8]:

$$
H=\int_{k \in \mathbb{T}^{d}} \oplus H(k) d \eta .
$$

Таким образом, дискретный оператор Шредингера $H(k)$ имеет вид [8]

$$
H(k)=H_{0}(k)-V, \quad k \in \mathbb{T}^{d} .
$$

Для каждого $k \in \mathbb{T}^{d}$ невозмущенный оператор $H_{0}(k)$ является оператором умножения на функцию

$$
\mathcal{E}_{k}(q)=\varepsilon_{1}(q)+\varepsilon_{2}(k-q)
$$

то есть

$$
\left(H_{0}(k) f\right)(q)=\mathcal{E}_{k}(q) f(q), \quad f \in L_{2}\left(\mathbb{T}^{d}\right),
$$

a $V$ - компактный интегральный оператор:

$$
(V f)(q)=\int_{\mathbb{T}^{d}} v(q-s) f(s) d \eta(s), \quad f \in L_{2}\left(\mathbb{T}^{d}\right) .
$$

Так как оператор возмущения $V$ компактный, из теоремы Вейля о сохранении существенного спектра следует, что существенный спектр $\sigma_{\text {ess }}(H(k))$ оператора $H(k)$ совпадает со спектром $\sigma\left(H_{0}(k)\right)$ невозмущенного оператора $H_{0}(k)$ [9], а именно

$$
\sigma_{\mathrm{ess}}(H(k))=\left[\mathcal{E}_{\min }(k), \mathcal{E}_{\max }(k)\right]
$$

где

$$
\mathcal{E}_{\min }(k) \equiv \min _{q \in \mathbb{T}^{d}} \mathcal{E}_{k}(q), \quad \mathcal{E}_{\max }(k) \equiv \max _{q \in \mathbb{T}^{d}} \mathcal{E}_{k}(q)
$$

и функция $\mathcal{E}_{k}(q)$ определена в $(1)$.

2.1. Условно отрицательно определенные функции. Напомним (см. монографию [9]), что комплекснозначная ограниченная функция $\varepsilon: \mathbb{T}^{d} \rightarrow \mathbb{C}$ называется условно отрицательно определенной, если $\varepsilon(p)=\overline{\varepsilon(-p)}$ и

$$
\sum_{i, j=1}^{n} \varepsilon\left(p_{i}-p_{j}\right) z_{i} \bar{z}_{j} \leqslant 0
$$

для любого $n \in \mathbb{N}$, любых $p_{1}, p_{2}, \ldots, p_{n} \in \mathbb{T}^{d}$ и вектора $\mathbf{z}=\left(z_{1}, z_{2}, \ldots, z_{n}\right) \in \mathbb{C}^{n}$, удовлетворяющего соотношению $\sum_{i=1}^{n} z_{i}=0$. 
Предположим, что дисперсионное соотношение $\varepsilon(p)$ является вещественнозначной непрерывной условно отрицательно определенной функцией, тогда $\varepsilon(p)$ - четная функция, которая имеет минимум в точке $p=0$.

Сделаем следующие предположения.

УСловиЕ 1. Функиия $\mathcal{E}_{k}(q)$, задающая дисперсионное соотношение двух частии, имеет следующий вид:

$$
\mathcal{E}_{k}(q)=m_{1} \varepsilon(q)+m_{2} \varepsilon(k-q), \quad m_{1}, m_{2}>0,
$$

где $\varepsilon(p)$ есть вещественнозначная дважды непрерывно дифферениируемая функиия на $\mathbb{T}^{d}$, причем $\varepsilon(0)=\min \varepsilon(q)$ u $\varepsilon(\vec{\pi})=\max \varepsilon(q)$ суть ее единственные невырожденные минимум и максимум.

УсловиЕ 2. Функиия $\varepsilon(p)$ условно отрицательно определена на $\mathbb{T}^{d}$.

Условие 3. Функиия $\hat{v}(s)$ неотрицателъна при всех $s \in \mathbb{Z}^{d}$, а ее преобразование Фуръе $v(k)$ является непрерывной бункцией на $\mathbb{T}^{d}$.

\section{3. ПОНЯТИЕ РЕЗОНАНСА С ЭНЕРГИЕЙ $\mathcal{E}_{\min }(0)$}

Пусть $d \geqslant 3$. Предположим, что выполнены условия 1 и 3 , которые гарантируют некоторую гладкость дисперсионного соотношения $\varepsilon(p)$ и преобразования Фурье $v$ взаимодействия $\hat{v}$. Введем понятие резонанса с энергией $\mathcal{E}_{\min }(0)$ (порогового виртуального уровня) для оператора энергии $H(k)$.

Для любого $z \in \mathbb{R} \backslash \sigma_{\mathrm{ess}}(H(k))$ определим самосопряженный компактный интегральный оператор $B(k, z)$ Бирмана-Швингера в гильбертовом пространстве $L_{2}\left(\mathbb{T}^{d}\right)$ формулой

$$
B(k, z)=V^{1 / 2} R_{0}(k, z) V^{1 / 2}
$$

где $R_{0}(k, z)$ - резольвента оператора $H_{0}(k)$ и $V^{1 / 2}$ - положительный корень из положительного оператора $V$, определенного в $(2)$.

Заметим, что при $z=\mathcal{E}_{\min }(0)$ оператор $R_{0}(k, z)$ не существует как ограниченный оператор, определенный во всем гильбертовом пространстве $L_{2}\left(\mathbb{T}^{d}\right)$. Однако, так как функция $\mathcal{E}_{0}(q)$ имеет невырожденный минимум $\mathcal{E}_{\min }(0)$ в точке $q=0$, можно определить следующую непрерывную функцию $B\left(p, q ; 0, \mathcal{E}_{\min }(0)\right)$ переменных $p \in \mathbb{T}^{d}$ и $q \in \mathbb{T}^{d}$ на $\left(\mathbb{T}^{d}\right)^{2}:$

$$
B\left(p, q ; 0, \mathcal{E}_{\min }(0)\right)=(2 \pi)^{-3} \int_{\mathbb{T}^{d}} \frac{v^{1 / 2}(p-t) v^{1 / 2}(t-q)}{\mathcal{E}_{0}(t)-\mathcal{E}_{\min }(0)} d t,
$$

где

$$
v^{1 / 2}(p)=(2 \pi)^{-3 / 2} \sum_{s \in \mathbb{Z}^{d}} \hat{v}^{1 / 2}(s) e^{i(p, s)}
$$

- ядро оператора $V^{1 / 2}$, а $\hat{v}^{1 / 2}(s)$ - положительный корень из положительной функции $\hat{v}(s)$. 
ЗАмЕчаниЕ 1 . Ясно, что число $z \leqslant \mathcal{E}_{0}(0)$ является собственным значением оператора $H(0)$ (т. е. $\operatorname{Ker}(H(0)-z I) \neq 0$ ), тогда и только тогда, когда единица является собственным значением компактного оператора $B(0, z)$, определенного в $L_{2}\left(\mathbb{T}^{d}\right)$, и существует функция $\psi \in \operatorname{Ker}(I-B(0, z))$ такая, что функция

$$
f(\cdot)=\frac{\left(V^{1 / 2} \psi\right)(\cdot)}{\mathcal{E}_{0}(\cdot)-z}
$$

принадлежит пространству $L_{2}\left(\mathbb{T}^{d}\right)$. В этом случае $f \in \operatorname{Ker}(H(0)-z I)$. Кроме того, если $z<\mathcal{E}_{\min }(0)$, то

$$
\operatorname{dim} \operatorname{Ker}(H(0)-z I)=\operatorname{dim} \operatorname{Ker}(I-B(0, z))
$$

и

$$
\operatorname{Ker}(H(0)-z I)=\left\{f \mid f(\cdot)=\frac{\left(v^{1 / 2} \psi\right)(\cdot)}{\mathcal{E}_{0}(\cdot)-z}, \psi \in \operatorname{Ker}(I-B(0, z))\right\} .
$$

В случае, когда $z=\mathcal{E}_{\min }(0)$ является собственным значением оператора $H(0)$, равенство (5) может оказаться неверным. Тогда его следует заменить на неравенство

$$
\operatorname{dim} \operatorname{Ker}(H(0)) \leqslant \operatorname{dim} \operatorname{Ker}\left(I-B\left(0, \mathcal{E}_{\min }(0)\right)\right) .
$$

ОПРЕДЕЛЕНИЕ 1 . Пусть $d \geqslant 3$. Точка $z=\mathcal{E}_{\min }(0) \in \sigma_{\mathrm{ess}}(H(0))$ называется регулярной точкой существенного спектра оператора $H(0)$, если единица не является собственным значением оператора $B\left(0, \mathcal{E}_{\min }(0)\right)$.

ОПРЕДЕЛЕНИЕ 2 . Пусть $d=3,4$. Будем говорить, что число $z=\mathcal{E}_{\min }(0)$ является резонансом с энергией $\mathcal{E}_{\min }(0)$ (пороговым виртуальным уровнем) оператора $H(0)$, если единица является простым собственным значением оператора $B\left(0, \mathcal{E}_{\min }(0)\right)$ и соответствующий собственный вектор $\psi$ удовлетворяет условию

$$
\frac{\left(v^{1 / 2} \psi\right)(\cdot)}{\mathcal{E}_{0}(\cdot)-\mathcal{E}_{\min }(0)} \in L_{1}\left(\mathbb{T}^{d}\right) \backslash L_{2}\left(\mathbb{T}^{d}\right),
$$

где $L_{1}\left(\mathbb{T}^{d}\right)$ - банахово пространство функций, определенных на $\mathbb{T}^{d}$.

ЗАмечАниЕ 2 . В случае $\mathcal{E}_{\min }(0)=0$ наше определение резонанса с энергией $\mathcal{E}_{\min }(0)$ для оператора $H(0)$ согласуется с определениями резонанса с нулевой энергией, ранее приведенными в работах [8], [10]-[12].

ЗАмечАниЕ 3 . Оператор $H(0)$ имеет резонанс с энергией $\mathcal{E}_{\min }(0)$ тогда и только тогда, когда единица является собственным значением оператора $B\left(0, \mathcal{E}_{\min }(0)\right)$ и хотя бы один (с точностью до нормировочного множителя) соответствующий собственный вектор $\psi \in L_{2}\left(\mathbb{T}^{d}\right)$ удовлетворяет условию

$$
\int_{\mathbb{T}^{d}} v^{1 / 2}(p) \psi(p) d p \neq 0 .
$$

ЗАмЕчАниЕ 4 . Пусть $d \geqslant 5$. Если единица является простым собственным значением оператора $B\left(0, \mathcal{E}_{\min }(0)\right)$, то число $z=\mathcal{E}_{\min }(0)$ является собственным значением оператора $H(0)$, так как в этом случае соответствующий собственный вектор $\psi$ удовлетворяет условию

$$
\frac{\left(v^{1 / 2} \psi\right)(\cdot)}{\mathcal{E}_{0}(\cdot)-\mathcal{E}_{\min }(0)} \in L_{2}\left(\mathbb{T}^{d}\right)
$$


другими словами,

$$
\operatorname{dim} \operatorname{Ker} H(0)+1=\operatorname{dim} \operatorname{Ker}\left(I-B\left(0, \mathcal{E}_{\min }(0)\right)\right) .
$$

Чтобы сформулировать аналогичные результаты для отталкивающих потенциалов, рассмотрим оператор

$$
H_{+}(k)=H_{0}(k)+V, \quad k \in \mathbb{T}^{d},
$$

где операторы $H_{0}(k)$ и $V$ те же, что и в определении оператора $H(k)$.

Введем понятие резонанса с энергией $\mathcal{E}_{\max }(0)$ (порогового виртуального уровня) для оператора энергии $H_{+}(k)$. Предположим, что выполнены условия 1 и 3 .

ОПРЕДЕЛЕНИЕ 3 . Пусть $d=3,4$. Будем говорить, что число $\mathcal{E}_{\max }(0)$ является резонансом с энергией $\mathcal{E}_{\max }(0)$ (пороговым виртуалъным уровнем) оператора $H_{+}(0)$, если число -1 является простым собственным значением оператора $B\left(0, \mathcal{E}_{\max }(0)\right)$ и соответствующий собственный вектор $\psi$ удовлетворяет условию

$$
\frac{\left(v^{1 / 2} \psi\right)(\cdot)}{\mathcal{E}_{0}(\cdot)-\mathcal{E}_{\max }(0)} \in L_{1}\left(\mathbb{T}^{d}\right) \backslash L_{2}\left(\mathbb{T}^{d}\right) .
$$

\section{4. ФОРМУЛИРОВКА ОСНОВНЫХ РЕЗУЛЬТАТОВ}

Основные результаты для оператора Шредингера $H(k), k \in \mathbb{T}^{d}, d \geqslant 3$, с притягивающим потенциалом взамодействия сформулируем в следующих теоремах. Теорема 1 утверждает, что если оператор $H(0)$ удовлетворяет условию $H(0) \geqslant \mathcal{E}_{\min }(0) I$, то для весьма широкого класса функций $\mathcal{E}_{k}(\cdot)$ и потенциалов взаимодействия $v$ справедливо неравенство $H(k)>\mathcal{E}_{\min }(0) I$, которое выполняется при всех ненулевых $k \in \mathbb{T}^{d}$. В теореме 2 утверждается, что для одного важного класса функций, а именно для условно отрицательно определенных функций, существуют собственные значения оператора Шредингера $H(k), k \in \mathbb{T}^{d}$, при дополнительном условии, что оператор $H(0)$ либо имеет резонанс с энергией $\mathcal{E}_{\min }(0)$, либо число $\mathcal{E}_{\min }(0)$ является его собственным значением.

Теорема 1. Пусть справедливо неравенство $H(0) \geqslant \mathcal{E}_{\min }(0) I$ и выполнены условия 1 , 3. Тогда при любом $k \in \mathbb{T}^{d} \backslash\{0\}$ оператор $H(k)$ удовлетворяет неравенству $H(k)>\mathcal{E}_{\min }(0) I$.

При исследовании двухчастичных дискретных операторов Шредингера обычно предполагается, что $\mathcal{E}_{\min }(0)=0$. Сформулируем соответствующий результат как следствие теоремы 1.

СлеДСТВИЕ 1. Пусть оператор $H(0)$ неотрицателъный, $H(0) \geqslant 0$, и выполнены условия 1, 3. Тогда при любом $k \in \mathbb{T}^{d} \backslash\{0\}$ оператор $H(k)$ строго положстельный, $H(k)>0$.

ТеОрема 2. Пусть справедливо неравенство $H(0) \geqslant \mathcal{E}_{\min }(0) I$, выполнены условия 1-3 и либо оператор $H(0)$ имеет резонанс с энергией $\mathcal{E}_{\min }(0) \quad($ ри $d=3,4)$, либо порог $z=\mathcal{E}_{\min }(0)$ существенного спектра оператора $H(0)$ является собственным значением (при $d \geqslant 5)$. Тогда при любом $k \in \mathbb{T}^{d} \backslash\{0\}$ справедливы следующие утверждения. 
1. Оператор $H(k)$ имеет хотя бы одно собственное значение $E(k)$, которое лежит левее порога $\mathcal{E}_{\min }(k)$ существенного спектра и удовлетворяет неравенству $E(k)>\mathcal{E}_{\min }(0)$.

2. Для любого собственного значения $E(k)$ оператора $H(k)$, лежсащего левее порога $\mathcal{E}_{\min }(k)$ существенного спектра, выполняется неравенство $E(k)>\mathcal{E}_{\min }(0)$.

ЗАмечАниЕ 5. Пусть выполняются условия теоремы 2. Тогда в некоторой области $G \subset \mathbb{T}^{d}$ при $k \in G$ оператор $H(k)$ может иметь несколько собственных значений, лежащих левее порога $\mathcal{E}_{\min }(k)$ существенного спектра. В силу теоремы 2 каждое из этих собственных значений удовлетворяет неравенству $E(k)>\mathcal{E}_{\min }(0)$. В частности, в случае $\mathcal{E}_{\min }(0)=0$, имеем $E(k)>0$.

Теперь сформулируем аналогичные результаты для оператора $H_{+}(k), k \in \mathbb{T}^{d}$, с отталкивающим взаимодействием.

Теорема 3. Пусть справедливо неравенство $H_{+}(k) \leqslant \mathcal{E}_{\max }(0) I$ и выполнены условия 1, 3. Тогда при любом $k \in \mathbb{T}^{d} \backslash\{0\}$ оператор $H_{+}(k)$ удовлетворяет неравенству $H_{+}(k)<\mathcal{E}_{\max }(0) I$.

ТЕОрема 4. Пусть справедливо неравенство $H_{+}(0) \leqslant \mathcal{E}_{\max }(0)$, выполнены условия 1-3 и либо оператор $H_{+}(0)$ имеет резонанс с энергией $\mathcal{E}_{\max }(0)($ при $d=3,4)$, либо порог $z=\mathcal{E}_{\max }(0)$ существенного спектра оператора $H_{+}(0)$ является собственным значением (при $d \geqslant 5)$. Тогда при любом $k \in \mathbb{T}^{d} \backslash\{0\}$ справедливы следующие утверждения.

1. Oператор $H_{+}(k)$ имеет хотя бъ одно собственное значение $E_{+}(k)$, которое лежит правее порога $\mathcal{E}_{\max }(k)$ существенного спектра и удовлетворяет неравенcmву $E_{+}(k)<\mathcal{E}_{\max }(0)$.

2. Любое собственное значение $E_{+}(k)$, лежащее правее порога $\mathcal{E}_{\max }(k)$ существенного спектра, удовлетворяет неравенству $E_{+}(k)<\mathcal{E}_{\max }(0)$.

ЗАмЕчАниЕ 6. Пусть выполняются условия теоремы 3 и $z=\mathcal{E}_{\max }(0)$ является регулярной точкой существенного спектра оператора $H(0)$. Тогда как следствие теоремы 3 получим, что $H_{+}(k)<\mathcal{E}_{\max }(0) I$ при любом $k \in \mathbb{T}^{d}$.

\section{5. СПЕКТРАЛЬНЫЕ СВОЙСТВА ДВУХЧАСТИЧНЫХ ОПЕРАТОРОВ $H(K)$ И ДОКАЗАТЕЛЬСТВА ОСНОВНЫХ РЕЗУЛЬТАТОВ}

Пусть $A$ - ограниченный самосопряженный оператор, действующий в гильбертовом пространстве $\mathcal{H}$, и $\lambda \in \mathbb{R}$. Обозначим через $N_{+}(\lambda, A)$ (через $N_{-}(\lambda, A)$ ) число собственных значений оператора $A$, больших (меньших) чем $\lambda$.

5.1. Принцип Бирмана-Швингера. Следующий вариант принципа Бирмана-Швингера для двухчастичных операторов Шредингера на решетке $\mathbb{Z}^{d}$ доказан в работе [13].

ТЕОРема 5. Справедливы следующие утверждения.

1. Если функиия $\phi \in L_{2}\left(\mathbb{T}^{d}\right)$ является решением уравнения $H(k) \phi=z \phi$, то функиця $\psi=V^{1 / 2} \phi \in L_{2}\left(\mathbb{T}^{d}\right)$ есть решение уравнения $\psi=B(k, z) \psi$, где оператор $B(k, z)$ задан в (3). 
2. Если функция $\psi \in L_{2}\left(\mathbb{T}^{d}\right)$ является решением уравнения $\psi=B(k, z) \psi$, то функция $\phi=\left[H_{0}(k)-z\right]^{-1} V^{1 / 2} \psi \in L_{2}\left(\mathbb{T}^{d}\right)$ есть решение уравнения $H(k) \phi=z \phi$.

3. Число z является собственным значением кратности $m$ для оператора $H(k)$ тогда и только тогда, когда единица является собственным значением кратносmи $m$ для оператора $B(k, z)$.

4. С учетом кратности число собственных значений оператора $H(k)$, менъших z, равно числу собственных значений оператора $B(k, z)$, больших единиць, т.е. имеет место равенство $\left.N_{-}(z, H(k))=N_{+}(1, B(k, z))\right)$.

Tеорема 6. Onератор $B(0, z)$ для $z \leqslant \mathcal{E}_{\min }(0)$ действует в $L_{2}\left(\mathbb{T}^{d}\right)$, принадлежит классу $\Sigma_{2}$ операторов Гильберта-Шмидта, положителен и слабо непрерывен на полуоси $(-\infty, 0]$, и выполняется равенство

$$
N_{-}\left(\mathcal{E}_{\min }(0), H(0)\right)=N_{+}\left(1, B\left(0, \mathcal{E}_{\min }(0)\right)\right) .
$$

ДоказАтельство. Функция $\mathcal{E}_{0}(q)$ имеет невырожденный минимум $\mathcal{E}_{\text {min }}(0)=0$ в точке $q=0$, следовательно, функция $B\left(p, q ; 0, \mathcal{E}_{\min }(0)\right)$, определенная по формуле (4), непрерывна и является квадратично-интегрируемой на $\left(\mathbb{T}^{d}\right)^{2}$, т. е. оператор $B\left(0, \mathcal{E}_{\min }(0)\right)$ принадлежит классу $\Sigma_{2}$ операторов Гильберта-Шмидта. Поэтому при всех $z \leqslant \mathcal{E}_{\min }(0)$ существует интеграл

$$
\int_{\mathbb{T}^{d}} \frac{\left|v^{1 / 2} \psi\right|^{2}}{\mathcal{E}_{k}(p)-z} d p
$$

Для любого $\psi \in L_{2}\left(\mathbb{T}^{d}\right)$ согласно равенству

$$
(B(0, z) \psi, \psi)=\int_{\mathbb{T}^{d}} \frac{\left|v^{1 / 2} \psi\right|^{2}}{\mathcal{E}_{0}(p)-z} d p
$$

функция $(B(0, z) \psi, \psi)$ непрерывна, положительна, монотонно возрастает при значениях $z \in\left(-\infty, \mathcal{E}_{\min }(0)\right]$ и ограничена. Поэтому, переходя к пределу под знаком интеграла Римана, получим для любой функции $\psi \in L_{2}\left(\mathbb{T}^{d}\right)$, что

$$
\begin{aligned}
\lim _{z \rightarrow \mathcal{E}_{\min }(0)}(B(0, z) \psi, \psi) & =\lim _{z \rightarrow \mathcal{E}_{\min }(0)} \int_{\mathbb{T}^{d}} \frac{\left|v^{1 / 2} \psi\right|^{2}}{\mathcal{E}_{0}(p)-z} d p= \\
& =\int_{\mathbb{T}^{d}} \frac{\left|v^{1 / 2} \psi\right|^{2}}{\mathcal{E}_{0}(p)-\mathcal{E}_{\min }(0)} d p=\left(B\left(0, \mathcal{E}_{\min }(0)\right) \psi, \psi\right) .
\end{aligned}
$$

Для любого $z<\mathcal{E}_{\min }(0)$ и любой $\psi \in L_{2}\left(\mathbb{T}^{d}\right)$ имеет место неравенство

$$
(B(0, z) \psi, \psi) \leqslant\left(B\left(0, \mathcal{E}_{\min }(0)\right) \psi, \psi\right) .
$$

Отсюда

$$
N_{+}(1, B(0, z)) \leqslant N_{+}\left(1, B\left(0, \mathcal{E}_{\min }(0)\right)\right) .
$$


В силу принципа Бирмана-Швингера (см. теорему 5) и неравенства (6) для любого $z<\mathcal{E}_{\min }(0)$ справедлива формула

$$
N_{-}(z, H(0))=N_{+}(1, B(0, z)) \leqslant N_{+}\left(1, B\left(0, \mathcal{E}_{\min }(0)\right)\right) .
$$

Функция $N_{-}(z, H(0))$ непрерывна в точке $\mathcal{E}_{\min }(0)$ слева, поэтому, переходя к пределу в (7) при $z \rightarrow \mathcal{E}_{\min }(0)$, имеем

$$
N_{-}\left(\mathcal{E}_{\min }(0), H(0)\right) \leqslant N_{+}\left(1, B\left(0, \mathcal{E}_{\min }(0)\right)\right) .
$$

Отсюда следует, что число $N_{-}\left(\mathcal{E}_{\min }(0), H(0)\right)$ конечно. В силу принципа Бирмана-Швингера и по определению числа $N_{-}(z, H(0))$ при любом $z<\mathcal{E}_{\min }(0)$ мы можем записать соотношение

$$
N_{+}(1, B(0, z))=N_{-}(z, H(0)) \leqslant N_{-}\left(\mathcal{E}_{\min }(0), H(0)\right) .
$$

Оператор $B(0, z)$ при $z \leqslant \mathcal{E}_{\min }(0)$ компактен и является непрерывным слева для $z \in\left(-\infty, \mathcal{E}_{\min }(0)\right]$, отсюда, переходя к пределу при $z \rightarrow \mathcal{E}_{\min }(0)$ в неравенстве $(8)$, получим, что

$$
N_{+}\left(1, B\left(0, \mathcal{E}_{\min }(0)\right)\right) \leqslant N_{-}\left(\mathcal{E}_{\min }(0), H(0)\right) .
$$

Неравенства (7) и (9) доказывают теорему.

5.2. Доказательство теорем 1 и 2. Напомним, что дисперсионное соотношение имеет следущий вид:

$$
\mathcal{E}_{k}(q)=m_{1} \varepsilon(q)+m_{2} \varepsilon(k-q), \quad m_{1}, m_{2}>0
$$

При выполнении условий теоремы 1 оператор $H(0)$ удовлетворяет неравенству $H(0) \geqslant \mathcal{E}_{\min }(0) I$. В силу предельного принципа Бирмана-Швингера заключаем, что для любого $\psi \in L_{2}\left(\mathbb{T}^{d}\right)$ имеет место следующее неравенство:

$$
\left(B\left(0, \mathcal{E}_{\min }(0)\right) \psi, \psi\right) \leqslant(\psi, \psi)
$$

По определению оператора Бирмана-Швингера

$$
\left(B\left(0, \mathcal{E}_{\min }(0)\right) \psi, \psi\right)=\int_{\mathbb{T}^{d}} \frac{\left|\left(V^{1 / 2} \psi\right)(q)\right|^{2}}{\mathcal{E}_{0}(q)-\mathcal{E}_{\min }(0)} d q .
$$

Следовательно,

$$
\int_{\mathbb{T}^{d}} \frac{\left|\left(V^{1 / 2} \psi\right)(q)\right|^{2}}{\mathcal{E}_{0}(q)-\mathcal{E}_{\min }(0)} d q \leqslant \int_{\mathbb{T}^{d}}|\psi(q)|^{2} d q
$$

где

$$
\left(V^{1 / 2} \psi\right)(q)=\int_{\mathbb{T}^{d}} v^{1 / 2}(q-p) \psi(p) d p
$$


В силу условий теоремы 1 имеем, что для любого $\psi \in L_{2}\left(\mathbb{T}^{d}\right)$ справедливо следующее неравенство:

$$
\int_{\mathbb{T}^{d}} \frac{\left|\left(V^{1 / 2} \psi\right)(q)\right|^{2}}{\left(m_{1}+m_{2}\right) \varepsilon(q)-\mathcal{E}_{\min }(0)} d q \leqslant \int_{\mathbb{T}^{d}}|\psi(q)|^{2} d q .
$$

Поскольку это неравенство выполнено для всех $\psi \in L_{2}\left(\mathbb{T}^{d}\right)$, получим соотношение

$$
\int_{\mathbb{T}^{d}} \frac{\left|\left(V^{1 / 2} \Phi_{k} \psi\right)(q)\right|^{2}}{\left(m_{1}+m_{2}\right) \varepsilon(q)-\mathcal{E}_{\min }(0)} d \eta \leqslant \int_{\mathbb{T}^{d}}\left|\left(\Phi_{k} \psi\right)(q)\right|^{2} d \eta,
$$

где $\Phi_{k}$ - оператор, определенный на $L_{2}\left(\mathbb{T}^{d}\right)$ формулой $\left(\Phi_{k} \psi\right)(q)=\psi(k+q)$.

Совершая замену переменной $p \rightarrow p-k$, имеем

$$
\left(V^{1 / 2} \Phi_{k} \psi\right)(q)=\int_{\mathbb{T}^{d}} v^{1 / 2}(q-p) \psi(k+p) d p=\int_{\mathbb{T}^{d}} v^{1 / 2}(q-p+k) \psi(p) d p=\left(V^{1 / 2} \psi\right)(q+k) .
$$

Учтем соотношения (10), (11) и, сделав замену переменной $q \rightarrow q-k$, получим

$$
\begin{aligned}
& \int_{\mathbb{T}^{d}} \frac{\left|\left(V^{1 / 2} \Phi_{k} \psi\right)(q)\right|^{2}}{\left(m_{1}+m_{2}\right) \varepsilon(q)-\mathcal{E}_{\min }(0)} d \eta=\int_{\mathbb{T}^{d}} \frac{\left|\left(V^{1 / 2} \psi\right)(q+k)\right|^{2}}{\left(m_{1}+m_{2}\right) \varepsilon(q)-\mathcal{E}_{\min }(0)} d \eta= \\
& =\int_{\mathbb{T}^{d}} \frac{\left|\left(V^{1 / 2} \psi\right)(q)\right|^{2}}{\left(m_{1}+m_{2}\right) \varepsilon(q-k)-\mathcal{E}_{\min }(0)} d \eta \leqslant \int_{\mathbb{T}^{d}}|\psi(k+q)|^{2} d \eta=\int_{\mathbb{T}^{d}}|\psi(q)|^{2} d \eta .
\end{aligned}
$$

Таким образом, справедливы следующие соотношения:

$$
\begin{gathered}
\int_{\mathbb{T}^{d}} \frac{\left|\left(V^{1 / 2} \psi\right)(q)\right|^{2}}{\left(m_{1}+m_{2}\right) \varepsilon(q)-\mathcal{E}_{\min }(0)} d \eta \leqslant \int_{\mathbb{T}^{d}}|\psi(q)|^{2} d \eta \\
\int_{\mathbb{T}^{d}} \frac{\left|\left(V^{1 / 2} \psi\right)(q)\right|^{2}}{\left(m_{1}+m_{2}\right) \varepsilon(q-k)-\mathcal{E}_{\min }(0)} d \eta \leqslant \int_{\mathbb{T}^{d}}|\psi(q)|^{2} d \eta
\end{gathered}
$$

ПрЕДЛОЖЕНИЕ 1. Для всех $m_{1}, m_{2}>0 u x, y>0$ выполненено следующее неравенство:

$$
\frac{m_{1}}{x}+\frac{m_{2}}{y} \geqslant \frac{\left(m_{1}+m_{2}\right)^{2}}{m_{1} x+m_{2} y}
$$

при этом равенство в (13) достигается тогда и только тогда, когда $x=y$.

ДоказАтельство. В известном неравенстве $x^{2}+y^{2} \geqslant 2 x y$ выбираем $x$ и $y$ следующим образом: $x=\left(m_{1} m_{2}\right)^{1 / 2} p$ и $y=\left(m_{1} m_{2}\right)^{1 / 2} q$, где $m_{1}, m_{2}>0$ и $p, q>0$. Тогда доказываемое утверждение следует из элементарных преобразований.

Пусть $0 \neq k \in \mathbb{T}^{d}$. Применяя неравенство (13) к функциям

$$
\varepsilon(q)-\frac{\mathcal{E}_{\min }(0)}{m_{1}+m_{2}}, \quad \varepsilon(q-k)-\frac{\mathcal{E}_{\min }(0)}{m_{1}+m_{2}},
$$


при почти всех $q \in \mathbb{T}^{d}$ имеем

$$
\begin{gathered}
\frac{m_{1}}{\varepsilon(q)-\mathcal{E}_{\min }(0) /\left(m_{1}+m_{2}\right)}+\frac{m_{2}}{\varepsilon(q-k)-\mathcal{E}_{\min }(0) /\left(m_{1}+m_{2}\right)}> \\
>\frac{\left(m_{1}+m_{2}\right)^{2}}{m_{1} \varepsilon(q)+m_{2} \varepsilon(q-k)-\mathcal{E}_{\min }(0)} .
\end{gathered}
$$

Из этого неравенства и неравенств (12) получаем

$$
\begin{aligned}
\int_{\mathbb{T}^{d}} & \frac{\left|\left(V^{1 / 2} \psi\right)(q)\right|^{2}}{m_{1} \varepsilon(q)+m_{2} \varepsilon(q-k)-\mathcal{E}_{\min }(0)} d \eta< \\
& <\int_{\mathbb{T}^{d}} \frac{1}{m_{1}+m_{2}}\left(\frac{m_{1}\left|\left(V^{1 / 2} \psi\right)(q)\right|^{2}}{\left(m_{1}+m_{2}\right) \varepsilon(q)-\mathcal{E}_{\min }(0)}+\frac{m_{2}\left|\left(V^{1 / 2} \psi\right)(q)\right|^{2}}{\left(m_{1}+m_{2}\right) \varepsilon(q-k)-\mathcal{E}_{\min }(0)}\right) d \eta= \\
= & \frac{m_{1}}{m_{1}+m_{2}} \int_{\mathbb{T}^{d}} \frac{\left|\left(V^{1 / 2} \psi\right)(q)\right|^{2}}{\left(m_{1}+m_{2}\right) \varepsilon(q)-\mathcal{E}_{\min }(0)} d \eta+ \\
& +\frac{m_{2}}{m_{1}+m_{2}} \int_{\mathbb{T}^{d}} \frac{\left|\left(V^{1 / 2} \psi\right)(q)\right|^{2}}{\left(m_{1}+m_{2}\right) \varepsilon(q-k)-\mathcal{E}_{\min }(0)} d \eta \leqslant \int_{\mathbb{T}^{d}}|\psi(q)|^{2} d \eta .
\end{aligned}
$$

Таким образом, имеет место следующее неравенство:

$$
\int_{\mathbb{T}^{d}} \frac{\left|\left(V^{1 / 2} \psi\right)(q)\right|^{2}}{m_{1} \varepsilon(q)+m_{2} \varepsilon(q-k)-\mathcal{E}_{\min }(0)} d \eta<\int_{\mathbb{T}^{d}}|\psi(q)|^{2} d \eta,
$$

из которого вытекает, что для любого $k \in \mathbb{T}^{d} \backslash\{0\}$ и любой $\psi \in L_{2}\left(\mathbb{T}^{d}\right)$ выполняется неравенство

$$
\left(B\left(k, \mathcal{E}_{\min }(0)\right) \psi, \psi\right)<(\psi, \psi) .
$$

Из этого неравенства и принципа минимакса заключаем, что все собственные значения оператора $B(k, 0)$ меньше единицы. Согласно принципу Бирмана-Швингера отсюда следует, что все собственные значения оператора $H(k), k \in \mathbb{T}^{d} \backslash\{0\}$, удовлетворяют неравенству $E(k)>\mathcal{E}_{\min }(0)$. Теперь доказательство первого утверждения теоремы 2 следует из результатов работы [8], а второе утверждение вытекает из доказанной выше теоремы 1.

Теоремы 3 и 4 доказываются аналогично теоремам 1 и 2.

Благодарности. Авторы выражают свою признательность рецензенту за ценные критические замечания. Работа поддержана Фондом фундаментальных исследований республики Узбекистан (грант № FA-F1-F079).

\section{Список литературы}

[1] С. Н. Лакаев, С. С. Улашов, ТМФ, 170:3 (2012), 393-408.

[2] K. Winker, G. Thalhammer, F. Lang, R. Grimm, J. Hesker Denschlog, A. J. Daley, A. Kantian, H. P. Büchler, P. Zoller, Nature, 441:7095 (2006), 853-856, arXiv: cond-mat/0605196.

[3] S. Albeverio, S. N. Lakaev, Z. I. Muminov, Ann. H. Poincaré, 5:4 (2004), 743-772.

[4] S. Albeverio, G. F. Dell Antonio, S. N. Lakaev, J. Phys. A, 40:49 (2007), 14819-14842, arXiv: math/0703191.

[5] М.Э. Муминов, ТМФ, 153:3 (2007), 381-387.

4 Теоретическая и математическая физика, т. 178, № 3, 2014 г. 
[6] S. Albeverio, S. N. Lakaev, A. M. Khalkhuzhaev, Markov Proc. Relat. Fields, 18:3 (2012), $387-420$.

[7] С. Н. Лакаев, Функ. анализ и его прил., 27:3 (1993), 15-28.

[8] S. Albeverio, S. N. Lakaev, K. A. Makarov, Z. I. Muminov, Commun. Math. Phys., 262:1 (2006), 91-115, arXiv: math-ph/0501013.

[9] М. Рид, Б. Саймон, Методъ современной математической физики, т. 4: Анализ операторов, Мир, М., 1982.

[10] A. V. Sobolev, Commun. Math. Phys., 156:1 (1993), 127-168.

[11] H. Tamura, Nagoya Math. J., 130 (1993), 55-83.

[12] Д. Р. Яфаев, Зап. научн. сем. ЛОМИ, 51 (1975), 203-216; D. R. Yafaev, Scattering Theory: Some Old and New Problems, Lecture Notes in Mathematics, 1735, Springer, Berlin, 2000.

[13] V. Bach, W. de Siqueira Pedra, S. Lakaev, Bounds on the discrete spectrum of lattice Schrödinger operators, Preprint mp-arc 10-143, University of Texas, Austin, 2011.

Поступила в редакцию 25.04.2013, после доработки 29.07.2013 